\title{
Higgs Bosons Searches at CDF
}

\author{
Cristóbal Cuenca Almenar \\ University of California Irvine, CA 92697, U.S.A. \\ (on behalf of the CDF Collaboration)
}

\begin{abstract}
.
Advanced analysis techniques together with increasing data samples are bringing the sensitivity of CDF to the Higgs boson very close to the SM predictions. These improvements translate into more stringent exclusions of parameter space in BSM Higgs sectors and of the SM mass range. The CDF Collaboration has a very active program on Higgs searches that comprises most accessible production mechanisms and decay channels in $p \bar{p}$ collisions at $\sqrt{s}=1.96 \mathrm{TeV}$. This contribution will also review the combination of the different channels, data samples and analysis techinques that currently produces one of the most exciting experimental results in our field.
\end{abstract}

Keywords: Higgs, Standard Model, SUSY, MSSM, CDF

PACS: 12.60.Fr Extensions of electroweak Higgs sector, 12.60.Jv Supersymmetric models, 14.80.Bn Standard-model Higgs bosons, 14.80.Cp Non-standard-model Higgs bosons

\section{INTRODUCTION}

Although the Higgs mechanism [1] was proposed in the 1960's, the fundamental particle it predicts, the Higgs boson $(h)$, has yet to be discovered. Direct limits from the LEP experiments exclude Higgs boson masses below $114.4 \mathrm{GeV} / \mathrm{c}^{2}$ [2] at $95 \%$ Confidence Level (CL), while electroweak precision measurements place an indirect upper limit on the mass of a SM Higgs boson of $154 \mathrm{GeV} / c^{2}$ [3] at 95\% CL.

Here we will summarize the status of the search for the Higgs bosons using the the CDF II detector [4] to analyze the $p \bar{p}$ collision data from the Fermilab Tevatron.

\section{SUMMARY OF STANDARD MODEL SEARCH EFFORTS}

For Higgs boson masses below $135 \mathrm{GeV} / c^{2}, b \bar{b}$ is the main decay mode [5]. For higher masses the Higgs boson decays primarily into a pair of $W$ bosons. Due to these different decay modes, it is natural to also split the discussion of the analysis effort at CDF into the low mass and high mass categories.

The search for a Higgs boson is quite challenging due to the large backgrounds and small signal expectation. In order to increase signal-background discrimination, analyses make maximal use of the information in each event by employing multivariate techniques to collect the discriminating power of multiple input variables into a single more powerful output variable.

The Higgs boson search strategy is to perform dedicated analyses for each distinct final state with a significant production rate and then combine these results to make a statement about the sensitivity of the CDF experiment to the SM Higgs boson. 


\section{Low mass Higgs boson searches}

In the $h \rightarrow b \bar{b}$ decay, each $b$ quark fragments into a jet of hadrons and the Higgs boson signal appears as a peak in the invariant mass distribution of these two jets. The two-jet signature alone is not useful to reject the dijet QCD background which is produced at the Tevatron with a rate about ten orders of magnitude higher than the Higgs boson. To handle this problem the low mass Higgs boson searches focus on production processes where the Higgs boson is produced in association with a $W$ or $Z$ boson $(V h)$.

The requirements of a high $P_{T}$ charged lepton candidate, missing transverse energy $\left(B_{T}\right)$, and at least one $b$-tagged jet reduce the background in the $W h \rightarrow \ell v b \bar{b}[6]$ search channel. Recently, CDF has introduced a new analysis which incorporates matrix element (ME) calculations into a boosted decision tree with previously used discriminants. The combined result is the most sensitive low mass analysis obtaining observed (expected) $95 \%$ C.L. limit of 5.3 (4.0) times the SM prediction of the production cross section for a Higgs boson mass of $115 \mathrm{GeV} / c^{2}$ using $4.3 \mathrm{fb}^{-1}$ of integrated luminosity..

The $Z h \rightarrow \ell \ell b \bar{b}$ [7] has a smaller background but also a smaller signal expectation do to the reduced cross section of $Z h$ production. The reach of this analysis has been improved by loosening the lepton identification requirements to increase signal acceptance. For a Higgs boson mass of $115 \mathrm{GeV} / \mathrm{c}^{2}$ the $\mathrm{NN}$ analysis obtains an observed (expected) 95\% C.L. limit of 5.9 (6.8) times the SM prediction of the production cross section using $4.1 \mathrm{fb}^{-1}$ of integrated luminosity.

The third major low mass Higgs analysis is based on a $E_{T}$ requirement and identifying $b$ jets but does not allow any reconstructed leptons in the events [8]. It is sensitive to $Z h \rightarrow v v b \bar{b}$ but also $W h \rightarrow \ell v b \bar{b}$ when the lepton escapes detection. Without the charged lepton requirement the dominant background is QCD events where mismeasured jets fake the $H_{T}$ requirement. The $B_{T}^{\prime}+b b$ analysis obtains observed (expected) 95\% C.L. limit of 6.1 (4.2) times the SM prediction of the production cross section for a Higgs boson mass of $115 \mathrm{GeV} / c^{2}$ using $3.6 \mathrm{fb}^{-1}$ of integrated luminosity.

\section{High mass Higgs boson searches}

At high mass the $h \rightarrow W W \rightarrow \ell v \ell v$ channel dominates the sensitivity to the Higgs boson. The leptonic decay mode of the $W$ bosons is chosen to reduce background and improve signal purity. The CDF analysis [9] includes all significant production modes (gluon fusion, $V h$, and vector boson fusion (VBF)), and splits the analysis up based on

the number of jets observed in the final state. This is useful since the background and signal composition change considerably depending on the number of jets. The $h \rightarrow W W$ analysis is the most sensitive single analysis at CDF, yielding an observed (expected) 95\% C.L. limit of 1.2 (1.2) times the SM prediction cross section for a Higgs boon mass of $160 \mathrm{GeV} / c^{2}$ using $4.8 \mathrm{fb}^{-1}$ of integrated luminosity. 


\section{Results}

The observed limits on the Higgs boson cross section in units of the SM prediction for all of the CDF analyses are shown in Fig. 1(b). These results are combined into a single limit on the Higgs boson production rate for each Higgs boson mass hypothesis, shown in Fig. 1(a). The limits range from 1.1 to about 5 times the prediction of the SM rate.
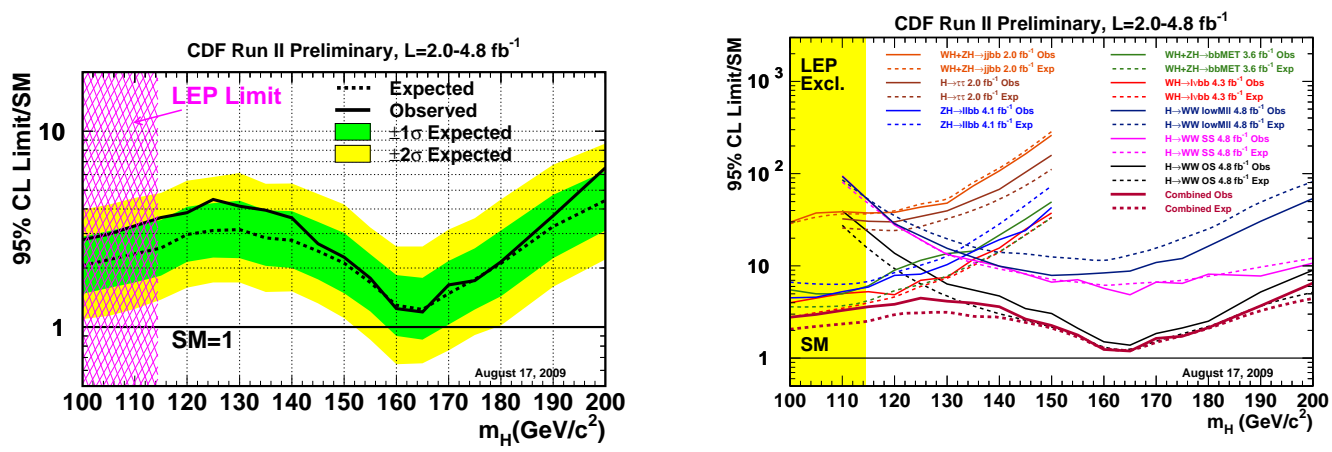

FIGURE 1. Limits at 95\% C.L. on the Higgs boson production cross section in factors away from the standard model prediction for the individual analysis channels (b) and the CDF combined result (a).

\section{BEYOND THE STANDARD MODEL HIGGS SEARCHES}

The CDF experiment also searches for Higgs bosons produced in several extensions to the SM, like the neutral and charged bosons of the Higgs sector of MSSM or the Higgs boson in a fermiofobic scenario.

Minimal Supersymmetric extension of the Standard Model (MSSM) [10] is a supersymmetric model with the minimal Higgs content, this is, with two Higgs doublets that provide five physical states. Three of this hypothesized boson are neutral ( $h, H$ and $A)$ while two of them are charged $\left(\mathrm{H}^{+}\right.$and $\left.\mathrm{H}^{-}\right)$. The masses of these boson are determined by two parameters, typically the mass of the pseudoscalar Higgs $m_{A}$ and $\tan (\beta)$, the ratio of the couplings to up-type and down-type quarks.

At the Tevatron, MSSM Neutral Higgs bosons would be produced through gluon fusion, mediated with a b-quark loop, and through b-quark annihilation. Due to the production enhancement factor of $\tan ^{2}(\beta)$, the Tevatron could have sensitivity to the high $\tan (\beta)$ region of the parameter space. The searches for this particles focuses on the main decay channels: two tau leptons or two b-quarks. Both of these decay channels have been probed by CDF. The results of the di-tau final state search, Fig. 2 pose now the most stringent results on this parameter space [11].

Another result that has been recently updated [12] is a search in the diphoton final state. The SM prediction for the $h \rightarrow \gamma \gamma$ branching fraction is extremely small (reaching a maximal value of only about $0.2 \%$ at a Higgs boson mass $\left.\left(m_{h}\right) \sim 120 \mathrm{GeV} / c^{2}\right)$ [5]; however, in "fermiophobic" models, where the coupling of the Higgs boson to fermions is suppressed, the diphoton decay can be greatly enhanced. This phenomenon has been shown to arise in a variety of extensions to the SM [13], and the resulting collider 
phenomenology has been described [14]. For this fermiophobic case, the decay into the diphoton final state dominates at low Higgs boson masses and is therefore the preferred search channel. The analysis results in $95 \%$ C.L. limits on the production cross section $(\sigma \times \mathscr{B} \mathrm{r}(h \rightarrow \gamma \gamma))$ and on the branching fraction $(\mathscr{B} \mathrm{r}(h \rightarrow \gamma \gamma)),[15]$.
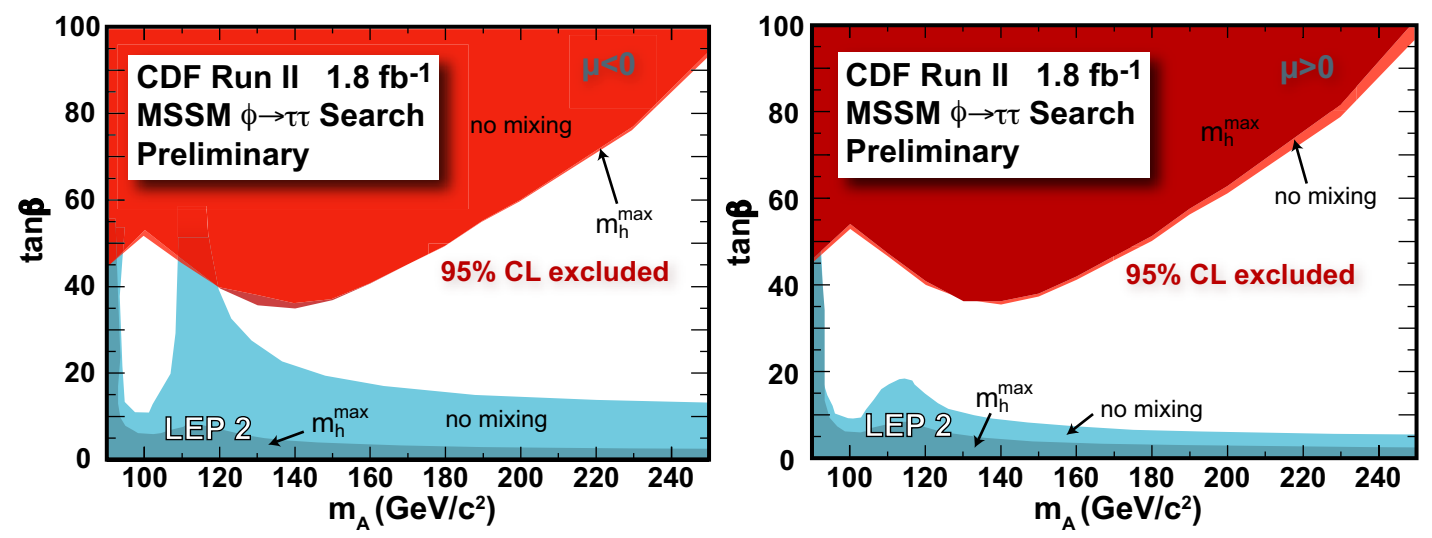

FIGURE 2. 95\% confidence level exclusion limits of the search for MSSM neutral Higgs bosons, interpreted in the $m_{\max }$ and no-mixing benchmark scenarios, for (a) negative and (b) positive $\mu$.

\section{CONCLUSIONS}

The CDF experiment is carefully searching for SM and BSM Higgs bosons. With more data on tape to analyze, and improvements still being added to analysis techniques, the Higgs boson search results will be an exciting topic until the end of the Tevatron run.

\section{REFERENCES}

1. Higgs P W 1964 Phys. Rev. Lett. 13 508-509

2. Barate R et al. (LEP Higgs Working Group) 2003 Phys. Lett. B 565 61-75

3. LEP-Tevatron-SLD Electroweak Working Group 2008 (Preprint arXiv : 0811.4682 )

4. Acosta D E et al. (CDF Collaboration) 2005 Phys. Rev. D 71032001

5. Djouadi A, Kalinowski J and Spira M 1998 Comput. Phys. Commun. 108 56-74

6. Aaltonen T et al. (CDF Collaboration) 2009 Phys. Rev. Lett. 103101802

7. CDF Collaboration, public note 9889

8. Aaltonen T et al. (CDF Collaboration) 2008 Phys. Rev. Lett. 100211801

9. Aaltonen T et al. (CDF Collaboration) 2009 Phys. Rev. Lett. 102021802

10. D. H. J. Chung et al., Phys. Rep. 407, 1 (2005) and references therein

11. Aaltonen T et al. (CDF Collaboration) 2009 submitted to Phys. Rev. Lett. (Preprint arXiv:0906. 1014)

12. Aaltonen T et al. (CDF Collaboration) 2009 (Preprint arXiv: 0905.0413 )

13. Akeroyd A G 1996 Phys. Lett. B 368 89-95

14. Mrenna S and Wells J D 2001 Phys. Rev. D 63015006

15. CDF Collaboration, public note 9586 